\title{
Assessing the Effects of Heavy Metal Concentration in Stream Sediments Within Onne and Environs Southeastern Nigeria
}

\author{
Omoko Ejiro Newton Ahiarakwem Cosmas Ahamefula Ibeneme Sabinus Ikechukwu \\ Onyekuru Samuel Okechukwu Nwankwor Godwin Ifedilichukwu \\ Department of Geology, Federal University of Technology Owerri
}

\begin{abstract}
This study assessed the seasonal variations in heavy metal concentration of stream sediments in parts of Onne, Rivers state Southeastern Nigeria. River Ogu was mapped for the collection of stream sediments because of its prominence as a receptor of a lot of effluents discharge from the NNPC oil refinery, erosion and weathering of heavy metals from nearby towns. Stream sediment samples were obtained from eleven (11) strategic locations at $3 \mathrm{~km}$ interval and; a control sample was obtained from Akpajo river to cover both the dry and wet seasons. Samples were analyzed with Atomic Absorption spectrometric method (AAS) and X-ray fluorescence spectroscopy (XRF) for $\mathrm{Ni}, \mathrm{Cu}, \mathrm{Hg}, \mathrm{Pb}, \mathrm{Zn}, \mathrm{Cd}$, As and $\mathrm{Cr}$. The record of the relative abundance of the heavy metals in the stream sediments in decreasing order for both seasons is as follows; Zinc $(\mathrm{Zn})>$ Nickel $(\mathrm{Ni})>$ Cadmium $(\mathrm{Cd})>$ Copper $(\mathrm{Cu})>$ Chromium $(\mathrm{Cr})>$ Mercury $(\mathrm{Hg})>$ Lead $(\mathrm{Pb})>$ Arsenic $(\mathrm{As})$. Higher mean concentration values were generally recorded in the wet season than in dry season. Four elements $(\mathrm{Zn}, \mathrm{Cd}, \mathrm{Ni}$ and $\mathrm{Cu})$ were found to be far higher than tolerable standard of the Federal Ministry of Environment (FME, 2006). The sources of the heavy metals are attributed to Onne refinery, Onne waste dump, runoff from roadside soil, acidic precipitation and effluents from petrochemical industries. Excessive concentrations of heavy metals in the stream sediments affects the aquatic life of the faunas and floras and have adverse effect on humans and this calls for regular monitoring of the chemistry of stream sediments in the area.
\end{abstract}

Keywords: Heavy metal, stream sediments, Atomic Absorption Spectrometry (AAS), x-ray fluorescence spectroscopy (XRF), River Ogu, Onne .

DOI: $10.7176 / \mathrm{JEES} / 9-10-05$

Publication date:October $31^{\text {st }} 2019$

\section{Introduction}

There is paucity of information as regards heavy metal concentration on stream sediments in the area of study. However, a lot of work has been carried out on the physicochemical analysis of the study area. The present study is aimed at investigating the physicochemical characteristics of the stream sediments in the study area. It is a fact that excessive concentration of heavy metals that can cause adverse effects, except its effect on humans and aquatic life, can affect the quality of groundwater where there is a leak to the groundwater resources of the area.

Stream sediments are river bed materials commonly found as deposits under the water level. Stream sediments serve as reservoirs for heavy metals and minerals.

River sediments are a major potential sink for hydrophobic pollutants in the aquatic environment (Kreina et al., 2003; Schorer, 1997). The sediments are mainly sedimentary materials that must have undergone series of terrestrial and marine transportation through some weathering and erosion processes. Heavy metals from roadside runoffs and other anthropogenic sources (industrial, waste dump, sewage effluents from nearby residential areas) are washed into streams and tributaries contaminating both the surface water and the sediments that have settled under the water. These underwater sediments exhibit the capacity to store the various pollutants transported through the river course and also provide geologic information as may be required. Heavy metals enter the surroundings by natural means and through human activities. Various sources of heavy metals include soil erosion, natural weathering of the earth's crust, mining, industrial effluents, urban runoff, sewage discharge, insect or disease control agents applied to crops, and many others (Morais et al., 2012).

Heavy metals may enter into aquatic ecosystems from anthropogenic sources, such as industrial wastewater discharges, sewage wastewater, fossil fuel combustion and atmospheric deposition (Linnik and Zubenko, 2000; Campbell, 2001; Lwanga et al., 2003; El Diwani and El Rafie, 2008; Idrees, 2009).

A polluted air has negative effects on the precipitation that falls into water bodies, stream sediments and soils. The precipitation can alter the chemistry of the soil, which, in turn, affects plant growth and water quality in a number of ways. This change is attributed principally to increased emissions of oxides of nitrogen and sulfur and their conversion to nitric and sulfuric acids (Galloway et al., 1976).

Acid precipitation has a number of potential effects on terrestrial ecosystems including acidification of soils, altered nutrient supply, increased mobilization of aluminum and other shifts from acid sensitive to acid tolerant species of soil flora and fauna population, altered rates of decomposition of organics and nitrogen fixation (Jacobson et al., 1988).

The dumping of solid waste in uncontrolled landfills can cause significant impacts on the environment and 
human health (Dong et al. 2008). Of these wastes, the household (and similar commercial) wastes are expected to generate the highest amounts of landfill gas, strongly dominated by the main components, methane and carbon dioxide. Many municipal and industrial wastes are rich in trace toxic metals (Yusuf et al., 2003).

Soil contamination by heavy metals is of most important apprehension throughout the industrialized world (Hinojosa et. al., 2004). However, various studies have reported elevated concentrations of metals in stream sediments originating from landfill contamination sources. Studies of increased $\mathrm{Cu}, \mathrm{Pb}, \mathrm{Zn}, \mathrm{Cd}, \mathrm{Ag}$, and $\mathrm{Ba}$ concentrations in stream sediments from landfills and water treatment facilities have been reported (Mantei and Coonrod, 1989; Mantei and Foster, 1991; Goncalves et al., 2004).

The extent of heavy metals from industrial effluents, acid rain deposition falling directly on the stream or through roadside runoffs and other man-made activities can pollute the aquatic ecosystems to a very high degree thereby negatively affecting the ecological balance of faunas in that environment.

The main objective of this work is to evaluate the impact of heavy metal concentration in stream sedimenst in the study area and thereby provide proper management strategy for sustainable development in the area. Some of the specific objectives are (1) determine the concentration of major pollutants with a view to assessing their level of pollution in the stream sediments of Ogu river. (2) establish the physico-chemical parameters in stream sediments to ascertain its pollution status and (3) develop some geochemical models and access the geochemical facies of the study area.

Heavy metals are significant environmental pollutants and their toxicity is a problem

of increasing significance for ecological, evolutionary, nutritional and environmental reasons (Jaishankar et al., 2014; Nagajyoti et al., 2010). The most commonly found heavy metals in waste water include arsenic, cadmium, chromium, copper, lead, nickel, and zinc, all of which cause risks for human health and the environment (Lambert et

al., 2000). This study was conducted during April 2018 to February 2019, along river Ogu in Onne, Eleme Local Government Area of River State Southern Nigeria.

\section{Materials and Methods}

\subsection{Sample collection}

Eleven (11) strategic locations of $3 \mathrm{~km}$ interval was mapped out along the course of Ogu River for the collection of stream sediments while samples were collected from a control location along Akpajo river which is about $6 \mathrm{~km}$ away amounting to 12 samples (fig 1). The top $20 \mathrm{~cm}$ of the stream sediment samples was collected from each sampling station using the Eckman bottom sampler. The stream sediment samples were pre-treated before final analysis.

\subsection{Sample Treatment and Analysis}

All the samples of sediments collected were allowed to thaw and air-dried at ambient temperature, sieved through

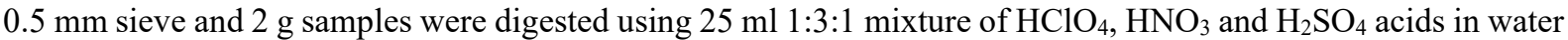
bath. A $10 \mathrm{ml}$ deionized water was added and decanted into $50 \mathrm{ml}$ standard flask and after rinsing, the solution was made up to the mark with deionized water. Buck Scientific atomic absorption spectrophotometer model 200A and air-acetylene flame were used for trace metal analyses. Accuracy of the method was checked using a standard sediment sample PACS- 2. For organic carbon, $1 \mathrm{~g}$ dried, sieved sediment was weighed into $250 \mathrm{ml}$ conical flask and digested with $10 \mathrm{ml} 0.5 \mathrm{M} \mathrm{K}_{2} \mathrm{Cr}_{2} \mathrm{O}_{7}$ and $20 \mathrm{ml}$ conc $\mathrm{H}_{2} \mathrm{SO}_{4}$, for $30 \mathrm{~min}$ (Walkey and Black, 1934). $100 \mathrm{ml}$ deionized water and 3 drops of Ferroin indicator were added and titrated with $0.25 \mathrm{M} \mathrm{FeSO}_{4}$ solution. Organic matter was obtained by multiplying organic carbon values by 1.724 .

\section{Results /Discussion}

The presence of anthropogenic sources of pollution in Onne area and its environs in Southeastern part of Nigeria has contributed to the contamination of the various aspects that constitute man's environment and most importantly as it pertains to this work, the stream sediments in river Ogu, in onne and its environs. The results acquired from an Atomic Absorption Spectrophotometry analysis of the stream sediments samples collected from river Ogu at $3 \mathrm{~km}$ interval from April to August 2018 and from October 2018 to February 2019, covering both seasons of 2018 through 2019 are presented in Tables 1 and 2. The tables illustrate a higher mean concentration of heavy metals during the wet season compared to the data recorded during the dry season.

The mean values of heavy metal concentration for $\mathrm{Zn}(11.6 \mathrm{mg} / \mathrm{kg}), \mathrm{Ni}(2.37 \mathrm{mg} / \mathrm{kg}), \mathrm{Cd}(1.42 \mathrm{mg} / \mathrm{kg}), \mathrm{Cu}$ $(1.13 \mathrm{mg} / \mathrm{kg}), \mathrm{Cr}(0.80 \mathrm{mg} / \mathrm{kg}), \mathrm{Hg}(0.66 \mathrm{mg} / \mathrm{kg}), \mathrm{Pb}(0.40 \mathrm{mg} / \mathrm{kg})$ and $\mathrm{As}(0.36 \mathrm{mg} / \mathrm{kg})$ recorded during the dry season are higher than the FME 2006 standards (fig 2 and Table 1) while the values recorded during the wet season are $\mathrm{Zn}(12.28 \mathrm{mg} / \mathrm{kg}), \mathrm{Ni}(2.51 \mathrm{mg} / \mathrm{kg}), \mathrm{Cd}(1.70 \mathrm{mg} / \mathrm{kg}), \mathrm{Cu}(1.23 \mathrm{mg} / \mathrm{kg}), \mathrm{Cr}(0.90 \mathrm{mg} / \mathrm{kg}), \mathrm{Hg}(0.76 \mathrm{mg} / \mathrm{kg}), \mathrm{Pb}$ $(0.50 \mathrm{mg} / \mathrm{kg})$ and As $(0.46 \mathrm{mg} / \mathrm{kg})$ which also record higher reading than the standard of the FME 2006) (figure 3 and Table 2). The general heavy metal data collected during the wet season is higher than the data obtained during the dry season. Although the heavy metal concentration of some elements are not very high above the tolerable 
limit but the concentration of $\mathrm{Zn}, \mathrm{Cd}, \mathrm{Ni}$ and $\mathrm{Cu}$ recorded during the dry and wet season are far above the Federal Ministry of Environment (FME, 2006)) standard. The most abundant element measured from the sediment was $\mathrm{Zn}$ with $11.16 \mathrm{mg} / \mathrm{kg}$ during the dry season and $12.16 \mathrm{mg} / \mathrm{kg}$ in the wet season and are far above the FME 2006 limit. Generally, the metals in the stream sediment followed the same order of increasing concentration in both dry and wet seasons: Zinc $(\mathrm{Zn})>\operatorname{Nickel}(\mathrm{Ni})>$ Cadmium $(\mathrm{Cd})>$ Copper $(\mathrm{Cu})>$ Chromium $(\mathrm{Cr})>\operatorname{Mercury}(\mathrm{Hg})>\mathrm{Lead}$ $(\mathrm{Pb})>$ Arsenic $(\mathrm{As})$. Several other metals analyzed were in the order Potassium $(\mathrm{K})>$ Calcium $(\mathrm{Ca})>\operatorname{Iron}(\mathrm{Fe})>$ Vanadium $(\mathrm{V})>$ Selenium (Se). The sample analyzed from the control point have lower mean concentration value except copper $(\mathrm{Cu})$ and Cadmium $(\mathrm{Cd})$ that shows the same mean concentration as recorded from the sampling locations.

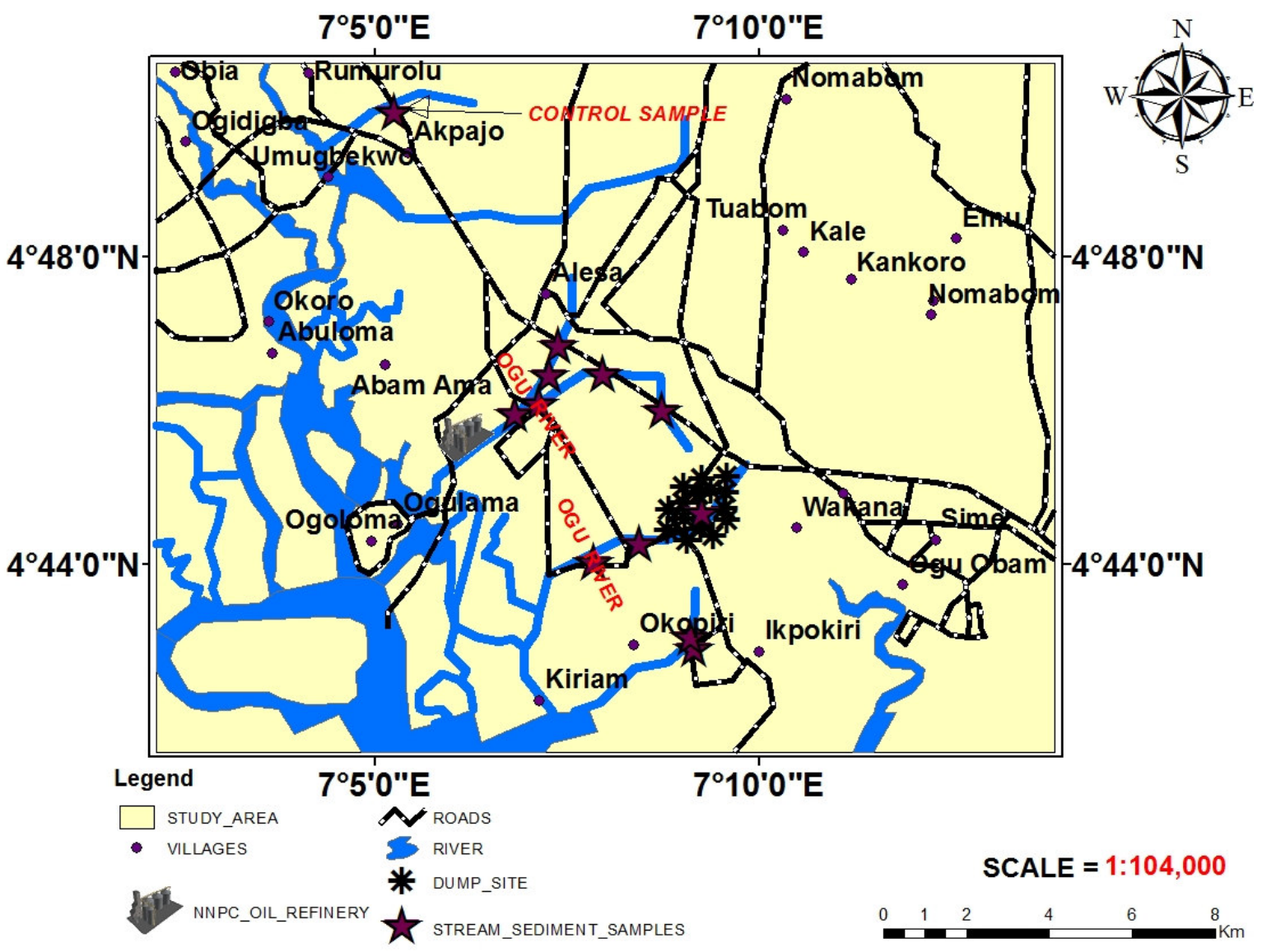

Fig.1: Location map showing stream sediment sampling location along Ogu river 
Table 1. Onne Stream Sediments (Dry Season)

\begin{tabular}{|c|c|c|c|c|c|c|c|c|c|c|c|c|c|c|}
\hline \multicolumn{13}{|c|}{ Gauge Stations } & \multirow{2}{*}{$\begin{array}{c}\text { Control } \\
\text { Point (3km } \\
\text { away) }\end{array}$} & \multirow{2}{*}{$\begin{array}{c}\text { FME } \\
2006 \\
\text { Standards } \\
\end{array}$} \\
\hline & 1 & 2 & 3 & 4 & 5 & 6 & 7 & 8 & 9 & 10 & 11 & \multirow[t]{2}{*}{ Mean } & & \\
\hline Coordinates & $\begin{array}{l}\mathrm{N} 4^{0} 42.9^{\prime} \\
\mathrm{E} 7^{0} 9.14^{\prime}\end{array}$ & $\begin{array}{l}{ }^{N} 4^{0} 43.03^{\prime} \\
\text { E } 7^{0} 9.12\end{array}$ & $\begin{array}{l}4^{0} 44.67^{\prime} \\
\text { E } 7^{0} 9.24^{\prime}\end{array}$ & $\begin{array}{c}\mathrm{N} \\
4^{0} 4.11 \\
\mathrm{E} 7^{0} 8.45^{\prime}\end{array}$ & $\begin{array}{l}\text { N } 4^{0} 44.03^{\prime}, \\
\text { E } 7^{0} 7.83^{\prime}\end{array}$ & $\begin{array}{l}\begin{array}{l}\mathrm{N} 4^{0} 46.01^{\prime} \\
\mathrm{E} 7^{0} 8.74^{\prime}\end{array}\end{array}$ & 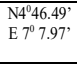 & $\begin{array}{l}\text { N4405.96' } \\
\text { E 7 } 6.82\end{array}$ & 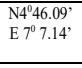 & 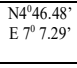 & 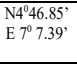 & & $\begin{array}{l}\mathrm{N}^{0} 49.9^{\prime} \\
\mathrm{E} 7^{0} 5.27^{\prime}\end{array}$ & \\
\hline $\begin{array}{c}\text { Elevation, } \mathrm{m} \\
\text { Parameters } \\
\end{array}$ & 7.92 & 9.14 & 24.30 & 19.20 & 19.50 & 21.33 & 21.64 & 16.76 & 18.28 & 19.50 & 18.89 & & 21.0 & \\
\hline $\mathrm{pH} @ 25^{0} \mathrm{C}$ & 6.22 & 6.24 & 6.26 & 6.28 & 6.24 & 6.22 & 6.26 & 6.28 & 6.20 & 6.20 & 6.24 & 6.24 & 6.40 & $6.5-8.5$ \\
\hline $\begin{array}{c}\begin{array}{c}\text { Electrical } \\
\text { conductivity, } \\
\text { pmhos } / \mathrm{cm}\end{array} \\
\end{array}$ & 694.90 & 70.00 & 74.00 & 695.50 & 69.60 & 72.00 & 75.70 & 76.20 & 68.30 & 74.00 & 69.05 & 185.38 & 190.30 & 1000 \\
\hline $\mathrm{Ca}, \mathrm{mg} / \mathrm{kg}$ & 10.00 & 12.60 & 8.80 & 9.60 & 10.00 & 10.50 & 11.82 & 8.80 & 10.60 & 12.00 & 11.80 & 10.59 & 60 & 200 \\
\hline $\mathrm{Na}, \mathrm{mg} / \mathrm{kg}$ & 12.68 & 12.00 & 10.80 & 12.83 & 11.80 & 12.00 & 12.40 & 11.00 & 12.00 & 11.60 & 12.60 & 11.97 & 80 & NS \\
\hline $\mathrm{Mg}, \mathrm{mg} / \mathrm{kg}$ & 5.50 & 5.80 & 5.60 & 5.40 & 5.20 & 5.0 & 5.02 & 5.04 & 5.80 & 5.82 & 5.84 & 5.45 & 5.00 & 100 \\
\hline $\mathrm{K}, \mathrm{mg} / \mathrm{kg}$ & 8.10 & 7.20 & 8.40 & 12.00 & 10.40 & 13.60 & 14.000 & 30 & 40 & 36 & 20 & 18.15 & 15 & 100 \\
\hline $\mathrm{Cl}, \mathrm{mg} / \mathrm{kg}$ & 80.50 & 82.00 & 70.00 & 75.00 & 76.50 & 70.00 & 73.30 & 84.60 & 82.00 & 71.00 & 65.00 & 75.44 & 100 & 250 \\
\hline $\mathrm{HCO}_{3}, \mathrm{mg} / \mathrm{kg}$ & 105.50 & 106.00 & 70.50 & 110.20 & 115.30 & 107.00 & 104.30 & 116.50 & 110.00 & 100.25 & 100.00 & 104.14 & 99.50 & NS \\
\hline $\mathrm{SO}_{4}, \mathrm{mg} / \mathrm{kg}$ & 38.50 & 37.00 & 34.30 & 35.00 & 40.00 & 30.25 & 32.00 & 45.50 & 42.20 & 28.30 & 25.80 & 35.35 & 27.50 & 200 \\
\hline $\mathrm{NO}_{3}, \mathrm{mg} / \mathrm{kg}$ & 76.00 & 76.50 & 60.20 & 80.50 & 82.20 & 76.00 & 75.80 & 85.80 & 76.80 & 70.00 & 68.80 & 75.32 & 65.90 & 50 \\
\hline $\begin{array}{c}\text { Total Iron, } \\
\mathrm{mg} / \mathrm{kg}\end{array}$ & 0.80 & 0.70 & 0.50 & 0.40 & 0.405 & 0.30 & 0.20 & 0.90 & 0.77 & 0.40 & 0.30 & 0.51 & 0.30 & \\
\hline $\mathrm{Ni}, \mathrm{mg} / \mathrm{kg}$ & 2.363 & 2.370 & 2.372 & 2.374 & 2.378 & 2.380 & 2.382 & 2.370 & 2.374 & 2.376 & 2.374 & 2.37 & 2.200 & 0.05 \\
\hline $\mathrm{Cu}, \mathrm{mg} / \mathrm{l}$ & 1.130 & 1.133 & 1.134 & 1.130 & 1.128 & 1.132 & 1.130 & 1.136 & 1.134 & 1.132 & 1.130 & 1.13 & 1.118 & 0.10 \\
\hline $\mathrm{Hg}, \mathrm{mg} / \mathrm{kg}$ & 0.668 & 0.670 & 0.672 & 0.666 & 0.664 & 0.662 & 0.660 & 0.658 & 0.656 & 0.654 & 0.652 & 0.66 & 0.0008 & 0.001 \\
\hline $\mathrm{Pb}, \mathrm{mg} / \mathrm{kg}$ & 0.425 & 0.426 & 0.428 & 0.426 & 0.424 & 0.422 & 0.420 & 0.386 & 0.384 & 0.382 & 0.380 & 0.40 & 0.330 & 0.05 \\
\hline $\mathrm{Zn}, \mathrm{mg} / \mathrm{kg}$ & 11.201 & 11.188 & 11.186 & 11.184 & 11.182 & 11.180 & 11.160 & 11.140 & 11.140 & 11.138 & 11.136 & 11.6 & 11.160 & 3.00 \\
\hline $\mathrm{Cd}, \mathrm{mg} / \mathrm{kg}$ & 1.433 & 1.435 & 1.431 & 1.430 & 1.428 & 1.426 & 1.424 & 1.420 & 1.421 & 1.420 & 1.422 & 1.42 & 1.380 & 0.05 \\
\hline $\mathrm{As}, \mathrm{mg} / \mathrm{kg}$ & 0.366 & 0.368 & 0.364 & 0.362 & 0.363 & 0.360 & 0.364 & 0.369 & 0.368 & 0.365 & 0.363 & 0.36 & 0.288 & 0.20 \\
\hline $\mathrm{Cn}, \mathrm{mg} / \mathrm{kg}$ & $\mathrm{ND}$ & $\mathrm{ND}$ & ND & $\mathrm{ND}$ & ND & $\mathrm{ND}$ & ND & $\mathrm{ND}$ & $\mathrm{ND}$ & $\mathrm{ND}$ & $\mathrm{ND}$ & 0.00 & ND & NS \\
\hline $\mathrm{Cr}, \mathrm{mg} / \mathrm{kg}$ & 0.808 & 0.806 & 0.805 & 0.810 & 0.811 & 0.803 & 0.802 & 0.800 & 0.802 & 0.802 & 0.788 & 0.80 & 0.700 & 0.05 \\
\hline $\mathrm{V}, \mathrm{mg} / \mathrm{kg}$ & 0.06 & 0.07 & 0.06 & 0.08 & 0.05 & 0.04 & 0.03 & 0.04 & 0.04 & 0.03 & 0.02 & 0.04 & 0.01 & 0.05 \\
\hline Se, $\mathrm{mg} / \mathrm{kg}$ & 0.04 & 0.05 & 0.06 & 0.04 & 0.04 & 0.03 & 0.02 & 0.04 & 0.05 & 0.06 & 0.04 & 0.04 & 0.01 & 0.05 \\
\hline $\begin{array}{c}\text { Oil and } \\
\text { grease, } \mathrm{mg} / \mathrm{kg}\end{array}$ & 0.02 & 0.02 & 0.03 & 0.03 & 0.04 & 0.03 & 0.02 & 0.02 & 0.03 & 0.03 & 0.02 & 0.025 & 0.006 & 5.00 \\
\hline $\mathrm{TPH}, \mathrm{mg} / \mathrm{kg}$ & 0.02 & 0.03 & 0.04 & 0.02 & 0.03 & 0.04 & 0.03 & 0.02 & 0.02 & 0.03 & 0.02 & 0.026 & 0.007 & NS \\
\hline BETEX,mg/kg & 0.059 & 0.058 & 0.062 & 0.064 & 0.066 & 0.064 & 0.062 & 0.060 & 0.062 & 0.060 & 0.060 & 0.061 & 0.050 & NS \\
\hline
\end{tabular}

Table 2. Onne Stream Sediments (Wet Season)

\begin{tabular}{|c|c|c|c|c|c|c|c|c|c|c|c|c|c|c|}
\hline \multicolumn{13}{|c|}{ Gauge Stations } & \multirow{2}{*}{$\begin{array}{c}\text { Control } \\
\text { Point (3km } \\
\text { away) } \\
\end{array}$} & \multirow{2}{*}{$\begin{array}{c}\text { FME } \\
2006 \\
\text { Standards } \\
\end{array}$} \\
\hline & 1 & 2 & 3 & 4 & 5 & 6 & 7 & 8 & 9 & 10 & 11 & Mean & & \\
\hline Coordinates & $\begin{array}{l}\mathrm{N}^{0} 42.9^{\prime} \\
\mathrm{E} 7^{0} 9.14^{\prime}\end{array}$ & $\begin{array}{l}4^{0} 43.03^{\prime} \\
\mathrm{E} 7^{0} 9.12^{\prime}\end{array}$ & $\begin{array}{l}\mathrm{N}^{0} 44.67^{\prime} \\
\mathrm{E} 7^{0} 9.24\end{array}$ & $\begin{array}{c}\mathrm{N} \\
4^{0} 44.11, \\
\mathrm{E} 7^{0} 8.45,\end{array}$ & $\begin{array}{l}\mathrm{N}^{\circ} 44^{0.03}, \\
\mathrm{E} 7^{0} 7.85^{\prime}\end{array}$ & $\begin{array}{l}4^{\circ} 46.01 \\
\text { E } 7^{0} 8.74^{\prime}\end{array}$ & $\begin{array}{l}4^{0} 46.49^{\prime} \\
\text { E } 7^{0} 7.97\end{array}$ & $\begin{array}{l}\mathrm{N}^{\circ} 45.96^{\prime} \\
\mathrm{E} 7^{0} 6.82^{\prime}\end{array}$ & $\begin{array}{l}\mathrm{N}^{0} 46.09^{\prime} \\
\mathrm{E} 7^{0} 7.14^{\prime}\end{array}$ & $\begin{array}{l}\mathrm{N}^{0} 46.48^{\prime} \\
\mathrm{E} 7^{0} 7.29^{\prime}\end{array}$ & $\begin{array}{l}\mathrm{N}^{0} 46.85^{\prime} \\
\mathrm{E} 7^{0} 7.39^{\prime}\end{array}$ & & $\begin{array}{l}4^{0} 49.9^{\prime} \\
\mathrm{E}^{0} 5.27\end{array}$ & \\
\hline $\begin{array}{c}\text { Elevation, } \mathrm{m} \\
\text { Parameters }\end{array}$ & 7.92 & 9.14 & 24.30 & 19.20 & 19.50 & 21.33 & 21.64 & 16.76 & 18.28 & 19.50 & 18.89 & & 21.0 & \\
\hline $\mathrm{pH} @ 25^{5} \mathrm{C}$ & 6.45 & 6.54 & 6.35 & 6.40 & 6.33 & 6.53 & 6.35 & 6.44 & 6.40 & 6.40 & 6.34 & 6.41 & 6.30 & $6.5-8.5$ \\
\hline $\begin{array}{l}\text { Electrical } \\
\text { conductivity, } \\
\text { umhos } / \mathrm{cm} \text {, }\end{array}$ & 696.90 & 85.00 & 84.00 & 699.50 & 89.60 & 82.00 & 85.70 & 86.20 & 78.30 & 84.00 & 75.05 & 195.11 & 200 & 1000 \\
\hline $\mathrm{Ca}, \mathrm{mg} / \mathrm{kg}$ & 11.00 & 12.60 & 10.80 & 10.60 & 11.00 & 10.50 & 11.82 & 13.80 & 12.60 & 13.00 & 14.80 & 12.04 & 60 & 00 \\
\hline $\mathrm{Na}, \mathrm{mg} / \mathrm{kg}$ & 14.68 & 13.00 & 13.80 & 13.83 & 12.80 & 15.00 & 14.40 & 13.00 & 12.00 & 12.60 & 14.60 & 13.61 & 80 & NS \\
\hline $\mathrm{Mg}, \mathrm{mg} / \mathrm{kg}$ & 5.80 & 5.95 & 5.65 & 5.60 & 5.95 & 5.30 & 5.10 & 5.09 & 6.10 & 5.98 & 6.04 & 5.68 & 5.20 & 100 \\
\hline $\mathrm{K}, \mathrm{mg} / \mathrm{kg}$ & 9.10 & 7.40 & 8.80 & 12.70 & 11.40 & 14.60 & 14.50 & 30.90 & 41.00 & 36.50 & 21.30 & 18.92 & 17 & 100 \\
\hline $\mathrm{Cl}, \mathrm{mg} / \mathrm{kg}$ & 85.50 & 87.00 & 74.00 & 77.00 & 79.50 & 73.00 & 75.30 & 87.60 & 85.00 & 74.00 & 68.00 & 78.71 & 100 & 250 \\
\hline $\mathrm{HCO}_{3}, \mathrm{mg} / \mathrm{kg}$ & 110.50 & 116.00 & 74.50 & 112.20 & 119.30 & 112.00 & 110.30 & 119.50 & 118.00 & 106.25 & 102.00 & 109.14 & 100.50 & NS \\
\hline $\mathrm{SO}_{4}, \mathrm{mg} / \mathrm{kg}$ & 40.50 & 39.00 & 37.30 & 38.00 & 44.00 & 35.25 & 38.00 & 46.50 & 47.20 & 33.30 & 29.80 & 38.98 & 30.50 & 200 \\
\hline $\mathrm{NO}_{3}, \mathrm{mg} / \mathrm{kg}$ & 79.20 & 78.50 & 65.00 & 84.50 & 86.20 & 78.00 & 80.80 & 88.80 & 80.80 & 73.00 & 72.80 & 78.87 & 69.90 & 50 \\
\hline $\begin{array}{l}\text { Total Iron, } \\
\mathrm{mg} / \mathrm{kg}\end{array}$ & 0.90 & 0.80 & 0.60 & 0.50 & 0.45 & 0.35 & 0.30 & 0.95 & 0.80 & 0.45 & 0.38 & 0.58 & 0.40 & \\
\hline $\mathrm{Ni}, \mathrm{mg} / \mathbf{k g}$ & 2.515 & 2.522 & 2.553 & 2.501 & 2.560 & 2.545 & 2.533 & 2.500 & 2.502 & 2.498 & 2.489 & 2.51 & 2.400 & 0.05 \\
\hline $\mathrm{Cu}, \mathrm{mg} / \mathrm{l}$ & 1.230 & 1.233 & 1.234 & 1.230 & 1.228 & 1.232 & 1.230 & 1.236 & 1.234 & 1.232 & 1.230 & 1.23 & 1.201 & 0.10 \\
\hline $\mathrm{Hg}, \mathrm{mg} / \mathrm{kg}$ & 0.768 & 0.770 & 0.772 & 0.766 & 0.764 & 0.762 & 0.760 & 0.758 & 0.756 & 0.754 & 0.752 & 0.76 & 0.001 & 0.001 \\
\hline $\mathrm{Pb}, \mathrm{mg} / \mathrm{kg}$ & 0.525 & 0.526 & 0.528 & 0.526 & 0.524 & 0.522 & 0.520 & 0.486 & 0.484 & 0.482 & 0.480 & 0.50 & 0.430 & 0.05 \\
\hline$\frac{\mathrm{Zn}, \mathrm{mg} / \mathrm{kg}}{\mathrm{Zng}}$ & $\frac{0.25}{13.201}$ & $\begin{array}{ll}12.188 \\
128\end{array}$ & $\frac{0.068}{12.186}$ & $\frac{0.200}{12.184}$ & $\frac{0.024}{12.182}$ & $\frac{1.222}{12.180}$ & $\frac{1.200}{12.160}$ & $\frac{0.400}{12.140}$ & $\frac{1.044}{12.340}$ & $\frac{1.02}{12.238}$ & $\frac{0.400}{12.136}$ & $\begin{array}{l}0.30 \\
12.28 \\
\end{array}$ & $\frac{1.400}{12.160}$ & $\frac{0.03}{3.00}$ \\
\hline $\mathrm{Cd}, \mathrm{mg} / \mathrm{kg}$ & 1.733 & 1.735 & 1.731 & 1.730 & 1.728 & 1.726 & 1.724 & 1.720 & 1.721 & 1.620 & 1.622 & 1.70 & 1.580 & 0.05 \\
\hline $\mathrm{As}, \mathrm{mg} / \mathbf{k g}$ & 0.466 & 0.468 & 0.464 & 0.462 & 0.463 & 0.460 & 0.464 & 0.469 & 0.468 & $\frac{1.020}{0.465}$ & 0.022 & $\begin{array}{l}1.10 \\
0.46\end{array}$ & 0.388 & 0.03 \\
\hline $\mathrm{Cn}, \mathrm{mg} / \mathrm{kg}$ & ND & ND & ND & ND & ND & ND & ND & ND & $\mathrm{ND}$ & ND & ND & 0.00 & ND & NS \\
\hline $\mathrm{Cr}, \mathrm{mg} / \mathrm{kg} / \mathrm{kg}$ & 0.908 & 0.906 & 0.905 & 0.910 & 0.911 & 0.903 & 0.902 & 0.900 & 0.902 & 0.902 & 0.888 & 0.90 & 0.800 & 0.05 \\
\hline $\mathrm{V}, \mathrm{mg} / \mathrm{kg}$ & 0.08 & 0.09 & 0.09 & 0.09 & 0.07 & 0.06 & 0.06 & 0.06 & 0.07 & 0.06 & 0.05 & 0.07 & 0.03 & 0.05 \\
\hline $\mathrm{Se}, \mathrm{mg} / \mathrm{kg}$ & 0.06 & 0.07 & 0.08 & 0.07 & 0.06 & 0.05 & 0.05 & 0.06 & 0.07 & 0.08 & 0.07 & 0.06 & 0.03 & 0.05 \\
\hline $\begin{array}{c}\text { Oil and } \\
\text { grease, } \mathrm{mg} / \mathrm{kg}\end{array}$ & 0.04 & $\begin{array}{l}0.07 \\
0.04\end{array}$ & 0.05 & 0.06 & 0.06 & 0.05 & 0.05 & 0.04 & 0.05 & 0.05 & 0.04 & 0.048 & 0.009 & $\frac{0.05}{5.00}$ \\
\hline $\mathrm{TPH}, \mathrm{mg} / \mathrm{kg}$ & 0.03 & 0.04 & 0.05 & 0.03 & 0.04 & 0.05 & 0.04 & 0.03 & 0.03 & 0.04 & 0.03 & 0.037 & 0.008 & NS \\
\hline 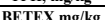 & 0.069 & 0.068 & 0.072 & 0.074 & 0.076 & 0.074 & 0.072 & 0.070 & 0072 & 0,070 & 0.070 & 0.071 & 0.060 & NS \\
\hline
\end{tabular}




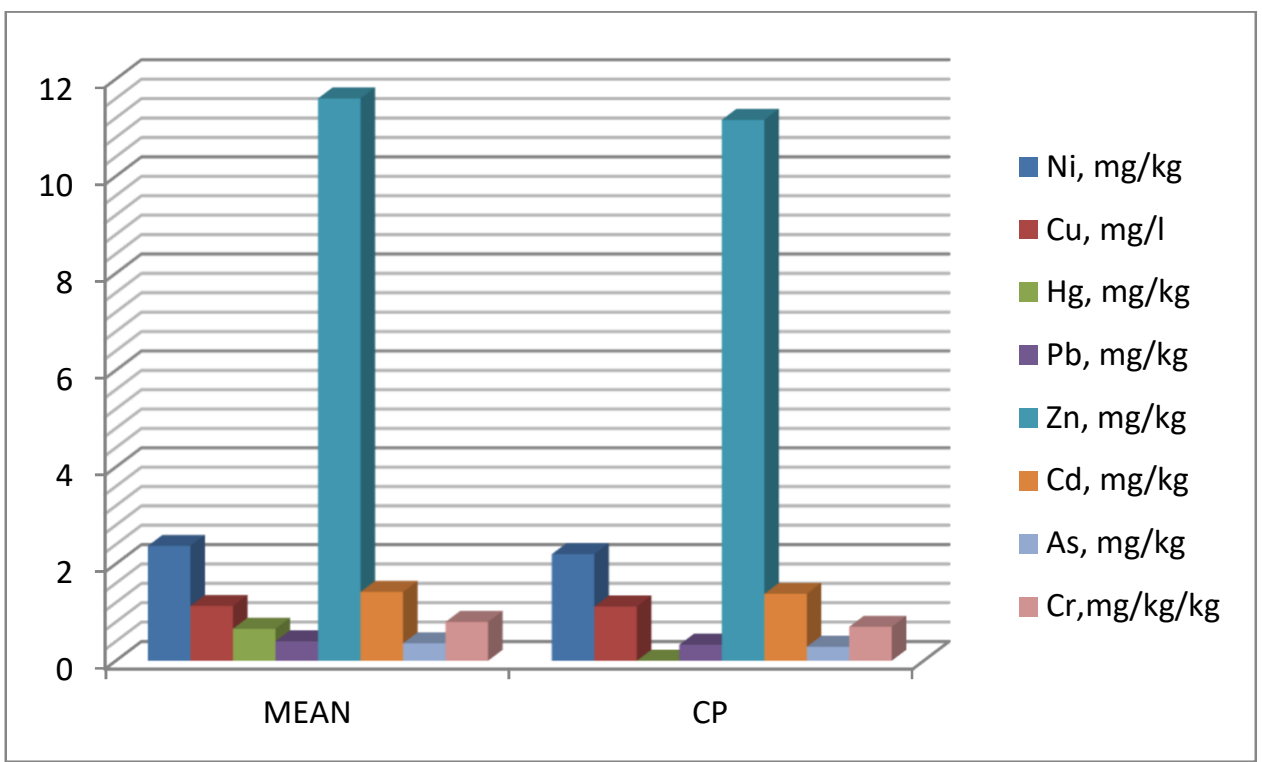

Fig. 2: Comparison of mean concentration of some heavy metals in stream sediments with the control point (Dry)

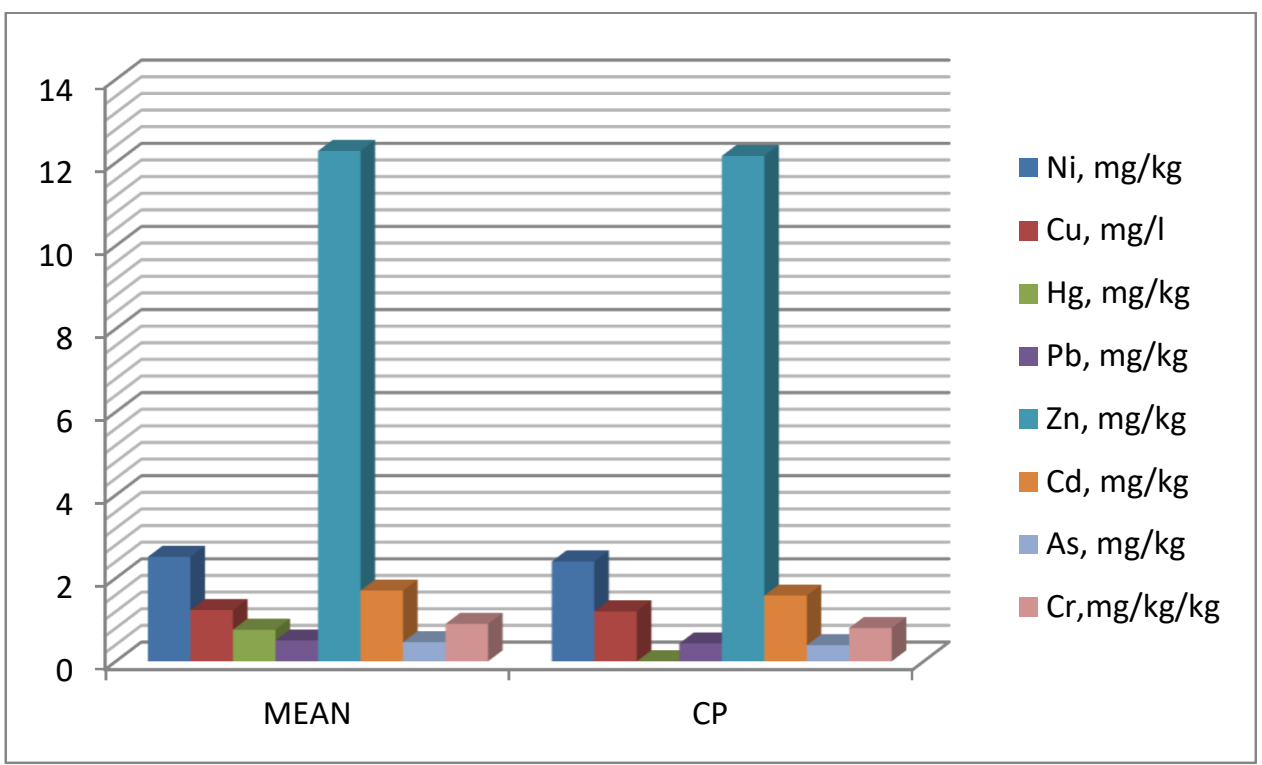

Fig 3: Comparison of mean concentration of some heavy metals in stream sediments with the control point (Wet)

\section{Conclusion}

The concentrations of heavy metals in stream sediment samples collected were analyzed and studied. The analysis carried out indicates that the concentration of $\mathrm{Zn}, \mathrm{Cd}, \mathrm{Ni}$ and $\mathrm{Cu}$ recorded during the dry and wet season are far above the Federal Ministry of Environment (FME, 2006) standard. The most abundant element measured from the sediment was $\mathrm{Zn}$ with $11.16 \mathrm{mg} / \mathrm{kg}$ during the dry season and $12.16 \mathrm{mg} / \mathrm{kg}$ in the wet season and are far above the FME (2006) limit. Heavy metal concentration in stream sediments is a serious issue most especially in the Niger Delta and Onne in particular hence to overcome this problem, substantial amount of research and development activities are needed as a key mitigation approach.

\section{References}

Campbell, L. M., (2001). Mercury in Lake Victoria (East Africa): Another emerging issue for a Beleaguered Lake? Ph.D. dissertation, Waterloo, Ontario, Canada.

Dong S, Liu B, \& Tang Z (2008) Investigation and modeling of the environment impact of landfill leachate on groundwater quality at Jiaxing Southern China. J Environ Technol Eng 1(1):23-30

El Diwani, G. \& El Rafie, Sh., (2008). Modification of thermal and oxidative properties of biodiesel produced from vegetable oils. Int. J. Environ. Sci. Tech., 5 (3), 391-400 (10 pages). 
FME (2006). Federal Ministry of Environment standard for soil.

Galloway J.N., Likens G.E \& Edgerton E.S.(1976). Acid precipitation in the northeastern United States: $\mathrm{pH}$ and acidity. Science, 194, :722-72

Goncalves, M.A., Nogueira, J.M.F, Figueiras,J., Putnis, C.V., and Almeida, C., 2004. Base-metals and organic content in stream sediments in the vicinity of a landfill, Applied Geochemistry, 19, 137-151.

Hinojosa M.B., Carreira J.A., Ruız R.G., \& Dick R.P. (2004). Soil moisture pre-treatment effects on enzyme activities as indicators of heavy metalcontaminated and reclaimed soils. Soil Biology \& Biochemistry, 36, $1559-1568$

Idrees, F. A., (2009). Assessment of trace metal distribution and contamination in surface soils of Amman. Jordan J. Chem., 4 (1), 77-87.

Jacobson J.S., L.I. Heller, K.E. Yamada, Osmeloski J.F.\& T. Bethard. (1988). Foliar injury and growth response of red spruce to sulphate and nitrate acidic mist. Canadian Journal of Forest Research, 20 :58-65.

Jaishankar M, Mathew BB, Shah MS, Gowda KRS. (2014). Biosorption of Few Heavy Metal Ions Using Agricultural Wastes. Journal of Environment Pollution and Human Health 2(1): 1-6.

Kreina A, Petticrew E, Udelhoven T (2003). The use of fine sediment fractal dimensions and colour to determine sediment sources in a small watershed. Catena 53:165-179.

Lambert M, Leven BA, Green RM. (2000). New methods of cleaning up heavy metal in soils and water. Environmental science and technology briefs for citizens. Kansas State University, Manhattan, KS.

Linnik, P. M.; Zubenko, I. B., (2000). Role of bottom sediments in the secondary pollution of aquatic environments by heavy metal compounds, lakes and reservoirs. Res. Manage., 5 (1), 11 - 21 (11 pages).

Lwanga, M. S.; Kansiime, F.; Denny, P.; Scullion, J., (2003). Heavy metals in Lake George, Uganda with relation to metal concentrations in tissues of common fish specie. Hydrobiologia, 499 (1-3), 83-93 (10 pages).

Morais S, Costa FG, Pereira ML. (2012). Heavy metals and human health, in Environmental health - emerging issues and practice (Oosthuizen J ed), pp. 227-246, InTech.

Nagajyoti PC, Lee KD, Sreekanth TVM. (2010). Heavy metals, occurrence and toxicity for plants: a review. Environ Chem Lett 8(3): 199-216.

Schorer M (1997). Pollutant and organic matter content in sediment particle size fractions Freshwater Contamination (Proceedings of Rabat Symposium S4, April-May 1997). IAHS Publ. 243:59.

Walkey A. \& Black LA (1934). An examination of the Dagtjareff method for determination of soil organic matter and a proposed modification of the chromic acid titration method. Soil Sci. 37:29-38.

Yusuf AA, Arowolo TA, Bamgbose O (2003). Cadmium, Copper and Nickel levels in vegetables from industrial and residential areas of Lagos City, Nigeria. Food Chem. Toxicol. 41:375378. 